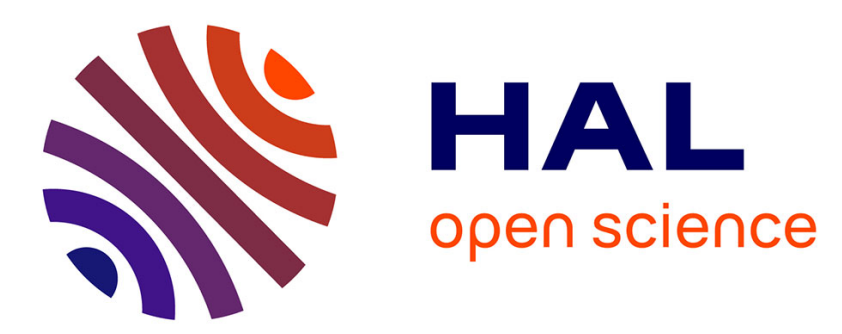

\title{
La mobilisation sanitaire des pouvoirs publics locaux : le cas de la ville de Wuhan
}

\author{
Jingyue Xing-Bongioanni, Jun Chu, Xuemin Qi
}

\section{To cite this version:}

Jingyue Xing-Bongioanni, Jun Chu, Xuemin Qi. La mobilisation sanitaire des pouvoirs publics locaux : le cas de la ville de Wuhan. Chronique internationale de l'IRES, 2020, Les mobilisations sanitaires des États et de l'Union européenne face à la première vague de Covid-19, 3 (171), pp.36-53. 10.3917/chii.171.0036 . halshs-03118461

\section{HAL Id: halshs-03118461 https://shs.hal.science/halshs-03118461}

Submitted on 16 Mar 2021

HAL is a multi-disciplinary open access archive for the deposit and dissemination of scientific research documents, whether they are published or not. The documents may come from teaching and research institutions in France or abroad, or from public or private research centers.
L'archive ouverte pluridisciplinaire HAL, est destinée au dépôt et à la diffusion de documents scientifiques de niveau recherche, publiés ou non, émanant des établissements d'enseignement et de recherche français ou étrangers, des laboratoires publics ou privés. 


\title{
Chine
}

\section{La mobilisation sanitaire des pouvoirs publics locaux : le cas de la ville de Wuhan}

\author{
Jing yue XING-BONGIOANNI, Jun CHU et Xuemin QI ${ }^{1}$
}

La pandémie de Covid-19 a placé la Chine au centre de l'attention des médias mondiaux. Selon les données publiées par la Commission nationale de santé (NHC) chinoise, au 29 février 2020, 79824 cas de contamination et 2870 morts avaient été enregistrés en Chine ${ }^{2}$. Différents reportages ont révélé des problèmes dans la gestion de la pandémie par le gouvernement chinois, portant notamment sur les mesures de censure. Les recherches en sciences sociales publiées en chinois se focalisent quant à elles sur deux thèmes $(\mathrm{He}, 2020$; Liu, Chen, Wang et Xia, 2020) : d'une part, le rôle des communautés de quartier (shequ) ${ }^{3}$ dans le gouvernement de la population urbaine pendant l'épidémie de Covid-19 ; d'autre part, la diffusion des informations sur Internet et le rôle des médias. Les travaux

1. Jingyue Xing-Bongioanni est chercheure postdoctorale au CEET, Cnam, et associée à Pacte (UMR 5194, CNRS/Université Grenoble Alpes/IEP de Grenoble) ; Jun Chu est doctorante en anthropologie culturelle à l'Institut d'anthropologie culturelle et d'ethnologie européenne à l'université de Göttingen, Allemagne ; Xuemin Qi est professeur d'histoire à Tsinghua University, Pékin, Chine.

2. La pandémie de Covid-19 n'a pas eu la même temporalité en Chine qu'en Europe. En Chine, le pic a été atteint début février 2020 mais depuis la mi-mars, le nombre de cas confirmés se maintient à un niveau très bas (moins de 100 nouveaux cas par jour sur toute la Chine). Source : http://www.nhc.gov.cn.

3. Les communautés de quartier (shequ) sont des « organisations autonomes de base des masses » créées en 1953 par la loi organisationnelle des comités de résidents de la Chine. Elles ont pour mission d'exercer un suivi social et de fournir des services sociaux de proximité aux habitants des quartiers concernés. Elles sont composées de bénévoles élus par les habitants locaux. La taille des communautés de quartier varie selon la ville et le district. Par exemple, la ville de Wuhan compte 13 districts, 156 sous-districts et 1316 communautés de quartier. Pour des analyses approfondies sur les communautés de quartier comme unités de gouvernement de la population urbaine en Chine, voir Bray (2006) et Audin (2015). 
publiés dans des revues en anglais ou en français ${ }^{4}$ se sont en revanche intéressés soit à la prise de décision par le gouvernement central chinois et au rôle joué par les experts scientifiques dans ce processus (Gu, Li, 2020 ; Liu, Zhong, Yu, 2020 ; Wei, 2020), soit aux expériences vécues par les habitants de Wuhan (Wang, 2020). Ces travaux ont surtout critiqué le déni et la censure du gouvernement chinois au début de l'épidémie, ou la non prise en compte des avis d'experts scientifiques dans la prise de décisions politiques.

Néanmoins, nous remarquons que les reportages et les travaux scientifiques existants sur l'épidémie de Covid-19 en Chine considèrent les gouvernements chinois central et locaux comme un tout. L'attention est principalement accordée au gouvernement central ${ }^{5}$ et peu de recherches ont tenu compte des rôles respectifs des gouvernements à différentes échelles et de leurs rapports durant la période de l'épidémie. Or, plusieurs chercheurs ont montré que la décentralisation, progressivement mise en place en Chine depuis $1978^{6}$, est plus développée qu'on ne pourrait le croire. Le déploiement des activités économiques, de la protection sociale (assurance maladie, retraite et assistance sociale), des soins, etc., relèvent de fait des compétences des gouvernements provinciaux, qui les financent sur la base des recettes fiscales locales et d'un transfert de recettes depuis le gouvernement central (Zhou, 2006, 2012). Dans ces domaines d'action publique, les gouvernements provinciaux négocient avec le gouvernement central et bénéficient de marges de manœuvre considérables dans la prise de décisions (Zhou, 2017).

Dans cet article, nous analysons les modalités de mobilisation sanitaire des pouvoirs publics chinois face à la pandémie de Covid-19, en les replaçant dans leur contexte de décentralisation partielle. Nous prenons la ville de Wuhan comme cas d'étude. Cette ville de 11 millions d'habitants est non seulement en effet le lieu où le premier cas de contamination est signalé, mais elle constitue également le véritable épicentre de l'épidémie, connaissant une croissance exponentielle de diffusion du virus entre début janvier et fin février 2020. Plus de $60 \%$ des cas de contamination et $76 \%$ des morts liés au Covid-19 en Chine y sont enregistrés ${ }^{7}$. En nous focalisant sur la gestion de la pandémie à Wuhan, nous

4. La revue Journal of Chinese Governance a publié un dossier consacré à la gouvernance de l'épidémie en Chine. Pour plus de précisions, voir « The COVID-19 Outbreak and Governing Public Health Emergency in China », Journal of Chinese Governance, vol. 5, n² 2, 2020, https:// www.tandfonline.com/toc/rgov20/5/2?nav=tocList.

5. Jusqu'en juillet 2020, un seul article a étudié les gouvernements locaux dans la lutte contre le Covid-19 en Chine. Mais l'analyse de ses deux auteurs (rattachés aux sciences de gestion), très normative, propose une série de recommandations de bonnes pratiques, ce qui ne permet pas d'appréhender le rôle des gouvernements locaux (Gao, Yu, 2020).

6. Depuis la proclamation de la République populaire de Chine en 1949, le pays a connu plusieurs réformes portant sur la structure politique, passant d'un pays extrêmement centralisé et d'une économie planifiée à un pays très décentralisé au plan économique. Les chercheurs chinois distinguent trois phases dans l'histoire de la décentralisation en Chine : de 1949 à 1978, la phase de centralisation totale ; de 1978 à 1994, la première phase de décentralisation et de 1994 à 2020, la seconde phase de décentralisation. Pour approfondir, voir Zhang (2011) et Jia et Zhao (2008).

7. En Chine, toutes les régions ont été touchées par la pandémie de Covid-19, mais le nombre de cas confirmés est resté très bas dans d'autres provinces : 1800 cas ont été enregistrés dans la province de Guangdong, 1282 cas à Zhejiang et 1279 cas à Henan, alors que la province de Hubei compte 68139 cas (dont 50338 à Wuhan). 
cherchons à comprendre les rôles respectifs des gouvernements central et locaux (provinciaux et municipaux), ainsi que les rapports qu'ils entretiennent durant cette période. Nous montrons que la gestion décentralisée de l'assurance maladie et de l'offre de soins a eu une forte influence sur les modalités de mobilisation sanitaire des pouvoirs publics face à la pandémie de Covid-19.

L'article se fonde sur une enquête qualitative par entretiens (téléphoniques/sur Zoom) menée entre avril et juin $2020^{8}$ ainsi que sur des études d'archives (documents publiés par les différentes administrations chinoises, dossiers transmis par nos enquêtés - documents de travail, captures d'écran des échanges dans les groupes de travail Wechat, etc.). Nous nous concentrons sur la période de début janvier à fin février en raison de la temporalité de l'épidémie en Chine : son pic est atteint début février, quand le nombre journalier de nouveaux cas de contamination dépasse 3500 , pour redescendre dès le 6 mars en dessous de 100 pour toute la Chine.

Afin de mieux contextualiser la mobilisation sanitaire de l'État chinois, nous présentons, dans la première partie, le système d'assurance maladie et l'offre de soins en Chine. Nous analysons dans les deux parties suivantes les mesures mises en place par les gouvernements chinois aux différentes échelles visant à augmenter la capacité d'accueil des hôpitaux et à permettre la gestion de l'afflux des patients du Covid-19. Dans la dernière partie, nous analysons la gestion de la pénurie de matériels médicaux et de protection, ainsi que la mobilisation de l'industrie par les gouvernements chinois.

\section{Un État central en retrait ? La gestion décentralisée de l'assurance maladie et de l'offre de soins}

En Chine, le système d'assurance maladie est construit assez récemment par rapport aux pays occidentaux ${ }^{9}$ : deux circulaires promulguées en 1951 et en 1952 introduisent une assurance maladie couvrant uniquement les citadins fonctionnaires et travailleurs d'entreprises nationales et collectives. Puis une assurance maladie couvrant l'ensemble de la population rurale, du nom de Nouvelle assurance maladie rurale coopérative, est introduite par une circulaire de 2003. Enfin, le Conseil des affaires de l'État publie en 2009 une autre circulaire qui crée une assurance maladie citadine pour les «non travailleurs » 10 : enfants, personnes âgées, chômeurs, étudiants, etc. Ce n'est qu'en 2010 qu'une véritable loi de Sécurité sociale voit le jour. Le système chinois d'assurance maladie est désormais composé de trois piliers : une branche pour les citadins travailleurs, une branche pour les citadins non travailleurs et une branche pour la population rurale.

8. Nos enquêtés comprennent un professeur d'une université pékinoise qui est conseiller spécial du maire de Pékin, trois médecins (dont un professeur d'une faculté de médecine à Wuhan, un médecin chef de pôle au Wuhan First Hospital et un médecin d'un service de réanimation pékinois ayant travaillé en renfort à Wuhan), un agent administratif d'un sous-district et un agent d'une communauté de quartier à Wuhan, ainsi que trois bénévoles responsables de deux groupes de solidarité créés pour soutenir Wuhan sur Wechat (équivalent de WhatsApp), en charge de récolter les dons (monétaires et en nature).

9. Pour une présentation plus précise du système d'assurance sociale maladie chinois, voir Meng et al. (2015).

10. http://www.gov.cn/zwgk/2009-04/07/content_1279256.htm. 


\section{Une assurance maladie différenciée selon les provinces, le statut et les branches}

L'assurance maladie est cofinancée par l'État et les provinces (sheng). Dans les dix dernières années, l'État central a publié plusieurs circulaires pour (re)définir le rôle du gouvernement dans le financement de l'assurance maladie à chaque échelle (Zhang, Li, 2015). Par exemple, le " plan de réforme relatif à la répartition des pouvoirs financiers et des responsabilités en matière de services publics entre le gouvernement central et les collectivités locales " ${ }^{11}$, mis en place le 8 février 2018, prévoit que les transferts financiers de l'État vers les départements varient selon la situation de l'économie et de la fiscalité locales. Cinq catégories ont ainsi été identifiées : dans celle des provinces « pauvres », l'État supporte $80 \%$ des dépenses de l'assurance maladie, et les provinces $20 \%$. À l'opposé, dans la catégorie des provinces riches, comprenant uniquement Pékin et Shanghai, qui sont considérées à la fois comme villes et provinces, la dotation de l'État ne représente que $10 \%$ du financement total de l'assurance maladie et les municipalités en financent la quasi-totalité. Ce plan confirme la décentralisation du financement et de la gestion de la couverture maladie.

Les modalités de cotisation et de remboursement de l'assurance maladie se différencient avant tout selon la branche (citadins travailleurs, citadins non travailleurs et ruraux), allant du simple au quadruple en termes de taux de remboursement. Plus précisément, nous identifions trois logiques de différenciation : d'abord se manifeste une logique de statut (hukou), c'est-à-dire une segmentation entre zones urbaines et rurales. Ensuite, le montant des cotisations et la générosité du système varient selon le secteur d'activité (public, privé) et la catégorie socioprofessionnelle. Enfin, les provinces bénéficient d'une grande marge de manœuvre dans la gestion de l'assurance maladie (en fixant des règles provinciales en termes de taux de prélèvement, de taux de remboursement, etc.).

\section{Une offre de soins dominée par le secteur hospitalier à la gestion décentralisée}

En ce qui concerne l'offre de soins, les ressources se concentrent dans le secteur hospitalier au détriment de soins ambulatoires qui s'effectuent notamment en cabinet médical. Par exemple, la notion de médecin traitant comme "gatekeeper », ou comme porte d'entrée du système de soins est introduite en 2011, qui fait l'objet d'une expérimentation volontaire à Shanghai. Ce n'est qu'en 2018 que l'Administration nationale de santé chinoise publie une circulaire encourageant le développement des médecins traitants. Jusqu'à présent, les Chinois n'ont pas besoin de passer par un médecin généraliste pour être soignés par un spécialiste à l'hôpital. Sauf en cas d'urgence, les Chinois peuvent se déplacer dans n'importe quel hôpital et faire la queue sur place, à la fois pour les maladies bénignes et pour les cas graves. Les travaux menés par certains chercheurs chinois ont montré que le choix du lieu de soin des adultes varie selon leurs caractéristiques sociodémographiques et socioéconomiques : plus un patient est jeune, diplômé, avec un revenu élevé, et plus il est probable qu'il se fasse soigner dans de meilleurs hôpitaux, notamment dans des

11. http://www.gov.cn/zhengce/content/2018-02/08/content_5264904.htm. 
établissements de santé classés en IIIA ${ }^{12}$. En revanche, les patients âgés, peu diplômés et au revenu relativement bas ont plus tendance à avoir recours aux centres de services sanitaires qui sont considérés comme des hôpitaux de proximité (Jiang, Yang, 2015). L'histoire du secteur hospitalier en Chine est marquée par une nationalisation entre 1949 et 1980, suivie par une lente privatisation à partir des années 1980 et par une accélération dans les années 2010 (Liu et al., 2014). En effet, le nombre d'hôpitaux privés à but lucratif ou non lucratif reste assez limité entre 1980 et 2007. Entre 2007 et 2010, un tournant politique dans la régulation publique du secteur hospitalier s'observe dans le rapport $\mathrm{du} 17^{\mathrm{e}}$ congrès national $\mathrm{du}$ Parti communiste chinois et dans plusieurs circulaires : le gouvernement encourage désormais le développement des établissements de santé privés, à but lucratif ou non. Au cours de la dernière décennie, le nombre d'hôpitaux privés est multiplié par trois et dépasse largement le nombre d'hôpitaux publics (tableau 1).

\section{Tableau 1. Évolution du nombre d'hôpitaux selon leur statut juridique (2010-2018)}

\begin{tabular}{|l|c|c|c|c|}
\hline Hôpitaux en Chine & \multicolumn{2}{|c|}{2010} & \multicolumn{2}{c|}{2018} \\
\hline & En nombre & En \% & En nombre & En \% \\
\hline Publics & 13850 & 66 & 12032 & 36 \\
\hline Privés à but non lucratif * & 1972 & 9 & 8419 & 26 \\
\hline Privés à but lucratif & 5096 & 25 & 12558 & 38 \\
\hline Ensemble & 20918 & 100 & 33009 & 100 \\
\hline
\end{tabular}

* En 1992, une circulaire émise par le ministère de la Santé autorise l'investissement des particuliers et des entreprises dans le secteur hospitalier en Chine. En 2000, une distinction entre hôpitaux privés à but lucratif et à but non lucratif est introduite par une circulaire publiée par le Conseil des affaires de l'État chinois. Les hôpitaux privés à but non lucratif en Chine, comme les établissements de santé privés à but non lucratif en France, ne distribuent aucun bénéfice aux fondateurs et gestionnaires. Ils bénéficient des avantages fiscaux et des subventions versées par les pouvoirs publics. Ces établissements, soumis à une tarification conjointe de l'administration locale de santé et de l'administration locale de l'industrie et du commerce jusqu'en fin d'année 2013 peuvent, depuis 2014, fixer librement leurs tarifs, à l'instar de leurs homologues privés à but lucratif. Source : Yearbook of health in the People's Republic of China, 2019.

Tout comme le financement et la gestion de l'assurance maladie, la régulation publique du secteur hospitalier n'est pas centralisée. Elle est confiée aux administrations de santé aux différents niveaux (ministériel, provincial et municipal). Ces administrations n'ont pas de rapports hiérarchiques entre elles : les

12. En Chine, les hôpitaux sont classés en dix catégories selon leurs domaines de compétence (soins généralistes, spécialistes, enseignement, recherche, etc.) et leur taille. Les hôpitaux IIIA, correspondant en France aux centres hospitaliers universitaires ( $\mathrm{CHU}$ ) et aux centres hospitaliers régionaux, représentent $8 \%$ des hôpitaux et sont majoritairement publics. Les centres de services sanitaires sont des établissements de santé de proximité, rattachés aux communautés de quartier. Selon la ville et le quartier, ces centres sont soit classés en catégorie I, soit non classés. 
administrations de santé provinciales et municipales sont respectivement soumises au pouvoir du gouverneur de province et du maire. Les hôpitaux publics sont encadrés par l'un de ces trois niveaux de l'administration. Par exemple, les tarifs d'un hôpital public provincial sont décidés conjointement par l'Administration de santé et l'Administration de l'industrie et du commerce provinciales, et ceux d'un hôpital public municipal par les autorités municipales (Li et al., 2020).

Le prix des médicaments est également encadré par le gouvernement chinois (Lian, 2019 ; Li, 2020). Jusqu'en 2015, les médicaments sont soumis à une tarification étatique par la Commission nationale du développement et de la réforme. Mais une nouvelle réforme introduite en 2015 supprime cette tarification. En 2020, seul le tarif des médicaments remboursés par l'assurance sociale maladie est encadré par les pouvoirs publics.

\section{Augmentation de la capacité d'accueil des hôpitaux et mobilisation des soignants}

La mobilisation sanitaire de l'État chinois dans la lutte contre le Covid-19 passe d'un extrême à l'autre : avant le 20 janvier 2020, les gouvernements à tous les niveaux sont dans le déni, censurant tout type d'alerte ; à partir du 20 janvier au contraire, sur les conseils d'un comité scientifique composé de professeurs de médecine ${ }^{13}$, ces mêmes gouvernements mettent en place de nombreuses mesures exceptionnelles et radicales comme la mise en quarantaine d'une ville entière, le confinement sévère des habitants et la construction de nouveaux établissements de santé pour accueillir les patients atteints du Covid-19.

Durant cette période, la lutte contre l'épidémie est beaucoup plus décentralisée qu'on ne pourrait le croire : les gouvernements provinciaux et municipaux jouent un rôle clé en prenant des décisions politiques importantes ; l'État central tient un rôle de coordonnateur entre les différents territoires et finance une part limitée des actions publiques.

\section{Augmenter en urgence la capacité d'accueil des hôpitaux}

La gestion de la capacité d'accueil des hôpitaux s'organise à l'échelle municipale. Prenons l'exemple de la ville de Wuhan. Le 20 janvier 2020, un Comité de direction municipal pour la lutte contre le Covid-19 (CDMLC) ${ }^{14}$ est mis en place par la mairie. Ce comité est composé du maire, du secrétaire du Comité du Parti pour la municipalité, du directeur de l'Administration municipale de santé, etc.

À Wuhan, un hôpital (Jin Yin Tan) est désigné pour accueillir les patients atteints de maladies contagieuses transférés par d'autres hôpitaux ou cliniques. Il est saturé dès le 20 janvier. Le 22 janvier, le CDMLC de Wuhan décide de transformer sept autres hôpitaux en points d'accueil des patients atteints du Covid-19. Ces hôpitaux sont surtout des CHU et d'autres centres hospitaliers relevant

13. Dans ce comité, composé de cinq professeurs en médecine, quatre faisaient partie du comité scientifique de lutte contre le syndrome respiratoire aigu sévère (Sras), une autre maladie infectieuse des poumons due à un coronavirus, en 2003. II est créé le 20 janvier par l'Administration nationale de santé, est élargi à 59 membres le 8 février et il comprend désormais de nouveaux membres de différents horizons (une quarantaine de chefs de pôle d'hôpitaux, quelques professeurs en santé publique, économistes et juristes).

14. Deux jours après, un comité de direction provincial, dirigé par le gouverneur et le secrétaire du Comité du Parti pour la province, est mis en place. 
de la catégorie III. Les patients initialement accueillis dans ces établissements sont transférés ailleurs ou renvoyés à leur domicile ${ }^{15}$. En une semaine, plus de 10000 lits sont mobilisés à Wuhan. Néanmoins, peu de patients peuvent être hospitalisés : selon les données communiquées par l'Administration municipale de santé, entre le 22 et le 27 janvier, plus de 75000 patients sont accueillis dans un des centres hospitaliers désignés, mais seuls 4000 d'entre eux sont hospitalisés, et continuent à dénoncer le manque de lits sur les réseaux sociaux.

Le CDMLC de Wuhan annonce la construction de deux nouveaux hôpitaux (Huoshenshan et Leishenshan) les 23 et 25 janvier. Il ne s'agit pas d'une mesure sans précédent. Ces deux hôpitaux reprennent en fait le plan de construction de l'hôpital Xiaotangshan, bâti en urgence à Pékin dans la lutte contre le Sras. Cette décision est soutenue par le Conseil des affaires de l'État avec un transfert de 500 millions de yuan (équivalant à 62,5 millions d'euros). Mais la construction de ces deux hôpitaux est majoritairement financée par les dons sociaux. En effet, la mairie de Wuhan mobilise 1,35 milliard de yuan (soit 169 millions d'euros) de dons récoltés par la Fédération de charité de Wuhan.

La construction de ces deux hôpitaux est réalisée en dix jours, ce qui impressionne le monde entier. Mais cela est rendu possible par le dur travail de plus de 40000 ouvriers de différents métiers issus d'entreprises publiques nationales (China Construction Third Engineering Bureau, Sinomach, etc.), ainsi que d'entreprises publiques municipales (Wuhan Iron and Steel, Wuhan Construction, etc.). Ces ouvriers ont dû annuler leur congé du Nouvel An chinois et reprendre le travail en urgence. Finalement, les hôpitaux Huoshenshan et Leishenshan, créant plus de 2600 lits de réanimation et de soins intensifs, pallient largement le manque de lits à Wuhan. Dans un dernier temps, le CDMLC cherche à augmenter la capacité des lieux d'accueil pour les patients présentant peu de symptômes ou des symptômes légers. Cet organisme est conseillé par le Pr. Chen Wang, professeur en pneumologie, vice-président de l'Académie chinoise d'ingénierie et anciennement président du comité scientifique dans la lutte contre le Sras en $2003{ }^{16}$. Le CDMLC annonce le 2 février la décision d'un confinement dans les centres collectifs des patients suspects ou atteints de Covid-19 afin de mieux maîtriser la propagation du nouveau coronavirus. Le lendemain, la mise en place d'hôpitaux de fortune (hôpitaux Fangcang) dans les locaux de stades, de centres d'exposition, etc., est annoncée par le CDMLC. Seize hôpitaux de fortune, cofinancés par les dons et le transfert du gouvernement central, sont ainsi construits en deux semaines et accueillent au total plus de 12000 patients.

15. Un mois après cette décision, le CDMLC publie une liste des hôpitaux accueillant désormais des patients atteints de maladies non liées au Covid-19. Néanmoins, selon notre enquêtée chef de pôle au Wuhan First Hospital, beaucoup de patients souffrant de maladies chroniques ont vu leur état de santé s'aggraver entre janvier et fin février. Plus de la moitié des décès dans les hôpitaux à Wuhan pendant cette période concernent les patients non atteints du nouveau coronavirus mais souffrant de maladies chroniques graves et qui n'ont pas été soignés ou mal soignés.

16. L'équipe de recherche du Pr. Wang a publié un article dans la revue The Lancet sur l'enjeu des hôpitaux Fangcang, ou hôpitaux de fortune (Chen et al., 2020). 


\section{Mobiliser les soignants}

La construction de nouveaux hôpitaux et la création de lits de réanimation et de soins intensifs se sont accompagnées d'une mobilisation des professionnels de santé (médecins et infirmiers) de grande ampleur. Néanmoins, les aides-soignants ne font pas l'objet de cette mobilisation : la plupart des hôpitaux accueillant des malades du Covid-19 suspendent en effet leurs postes. Les tâches qu'ils réalisaient initialement sont effectuées par les infirmiers pendant l'épidémie ${ }^{17}$. Deux types de mobilisation peuvent être identifiés : une mobilisation intra-régionale des professionnels de santé et une mobilisation interrégionale et des services de santé des armées.

Dans le premier cas, l'Administration municipale de santé demande dès le 20 janvier aux hôpitaux publics implantés à Wuhan de recenser le nombre de médecins et infirmiers volontaires pour travailler dans les hôpitaux dédiés aux patients atteints du Covid-19. Cependant, contrairement à la France, les étudiants en médecine et les professionnels retraités ne font pas l'objet de cette mobilisation. D'après notre entretien avec un médecin chef de pôle au Wuhan First Hospital, la quasi-intégralité des professionnels de santé de son service participent aux soins des patients atteints du Covid-19. Les médecins et infirmiers venant d'autres hôpitaux publics de Wuhan viennent en renfort pour assurer la continuité de service 24 heures sur 24 et 7 jours sur 7 . Une seconde vague de mobilisation est organisée par l'autorité locale de santé le 5 février, visant particulièrement les hôpitaux et cliniques privés à but lucratif ou non lucratif, afin de soulager le manque de professionnels. La répartition et l'envoi de 540000 médecins et infirmiers venant de 256 établissements de santé vers les 55 hôpitaux dédiés au Covid-19 sont gérés par l'Administration municipale de santé.

Dans le second cas, ces mobilisations des professionnels de santé sont coordonnées par l'Administration nationale de santé. À partir du 24 janvier, 280 équipes de renfort (35 000 professionnels dont un quart de médecins et trois quarts d'infirmiers) venant d'autres provinces chinoises s'installent dans différents hôpitaux dédiés aux patients contaminés à Wuhan. Selon notre enquête auprès d'un médecin pékinois venu en renfort à Wuhan entre fin janvier et fin février 2020, il y a eu plus de médecins volontaires que le nombre indiqué dans l'appel à renfort publié par son hôpital à Pékin. Cet hôpital pékinois a dû faire le tri et former une équipe d'« élite » comprenant notamment des médecins et infirmiers ayant eu l'expérience professionnelle de la lutte contre le Sras en 2003 et/ou ayant travaillé en soins intensifs et en réanimation. La mobilisation des services de santé des armées est plus fléchée : environ 4000 médecins et infirmiers de réanimation militaires sont déployés à Wuhan, notamment à l'hôpital Huoshenshan.

Deux types de mesures de récompense sont mises en place par le Conseil des affaires de l'État à partir du 22 février ${ }^{18}$. D'une part, les professionnels de santé ayant participé à la lutte contre le Covid-19 perçoivent une prime exceptionnelle de 300 yuan (soit 38 euros) par jour et les « salaires de base » sont

17. Cette décision est sans doute liée au développement du métier d'aide-soignant en Chine : aucun diplôme d'État correspondant n'a été créé. Les aides-soignants en poste sont en contrat précaire, recrutés par les hôpitaux ou employés directement par les patients.

18. http://www.gov.cn/gongbao/content/2020/content_5488912.htm. 
doublés pendant la période d'exercice. D'autre part, les médecins et infirmiers concernés bénéficient d'avantages dans l'évolution de leur carrière : la participation à la lutte contre le Covid-19 dans les hôpitaux leur permet d'être prioritaires dans les promotions et d'acquérir un an supplémentaire d'ancienneté. Ces mesures sont cofinancées par l'État central et les gouvernements locaux : l'État central ne finance que la prime journalière (300 yuan par jour). L'augmentation des salaires et les coûts éventuels liés aux avantages dans l'évolution de carrière sont pris en charge par les gouvernements municipaux et provinciaux.

Néanmoins, ces mesures sont critiquées par les médecins et infirmiers concernés. En effet, la circulaire prévoit que les professionnels perçoivent deux fois leur salaire de base comme rémunération. Or, dans les hôpitaux chinois, la rémunération des médecins est habituellement composée de trois parties : le salaire de base, une prime mensuelle d'activité (opérations, prescription d'examens, etc.) et une prime annuelle. Le salaire de base, fixé à l'échelle nationale en fonction du lieu d'exercice et du grade, reste très bas. Par exemple, le salaire de base mensuel d'un médecin en début de carrière se situe autour de 3000 yuan (environ 375 euros), et celui d'un professeur universitaire-praticien hospitalier en fin de carrière peut atteindre 9000 yuan (environ 1125 euros). En fonction du lieu d'exercice, de la spécialité et du grade, la prime d'activité d'un médecin peut correspondre à trois ou cinq fois son salaire de base. Selon notre enquête auprès d'un médecin du Wuhan First Hospital et d'un médecin pékinois ayant travaillé en renfort à Wuhan, les avantages monétaires promis par le gouvernement sont insuffisants par rapport à la perte des primes mensuelles d'activité pendant le Covid19. Au final, une grande partie des professionnels de santé perçoivent moins de revenus pendant la période de Covid-19 qu'avant.

\section{Gérer l'afflux de patients}

À Wuhan, bien que les pouvoirs publics aient augmenté la capacité d'accueil des hôpitaux en très peu de temps, l'inaction des gouvernements locaux et central pendant les trois premières semaines de janvier 2020 contribue à la propagation du virus. Le nombre de patients augmente exponentiellement. Dès le 23 janvier, le CDMLC met en place des mesures de confinement assez strictes visant à ralentir la propagation du virus (voir encadré), mais le manque cruel de lits hospitaliers et la difficulté d'accès aux soins pendant le Covid-19 durent pendant un mois (de mi-janvier à mi-février).

\section{Une distribution administrative des ressources rares}

La tension autour du manque de lits s'est concentrée sur l'accès aux soins intensifs et de réanimation. En effet, le CDMLC de Wuhan fixe une règle dès le 24 janvier : seuls les patients testés positifs au Covid-19 peuvent être hospitalisés. Autrement dit, à partir de cette date, bien que les patients puissent encore venir faire la queue à l'hôpital pour réaliser des examens (tomodensitométrie ou CT-Scan, bilan sanguin, etc.), ils ne peuvent plus être hospitalisés sans avoir obtenu les résultats du test PCR, même pour les cas très graves ou les personnes mourantes. L'admission en soins intensifs ou en réanimation n'est plus décidée par les médecins en fonction de la gravité des cas et de la disponibilité des lits. 


\section{Encadré}

\section{Les mesures de confinement à Wuhan}

Une série de mesures de confinement sont mises en place par le CDMLC entre fin janvier et début février à Wuhan afin de ralentir la propagation du virus.

\section{Phase 1 : la mise en quarantaine de la ville et la suspension des moyens de transports}

À la suite d'une décision du Conseil des affaires de l'État chinois appliquant la loi relative aux maladies infectieuses, la ville de Wuhan est mise en quarantaine à partir du 23 janvier à 10 heures : les stations ferroviaires, les aéroports ainsi que les autoroutes sont fermés. Les transports publics intra-muros sont interrompus. Le lendemain, $50 \%$ des taxis de la ville de Wuhan sont réquisitionnés et mis à disposition des communautés de quartier pour transporter les patients à l'hôpital. L'usage de voitures personnelles est interdit le 26 janvier. Cette série de mesures limite largement les déplacements des habitants.

\section{Phase 2 : le confinement des habitants dans leur quartier résidentiel ${ }^{1}$}

Le 11 février, le CDMLC annonce une nouvelle mesure restrictive en confinant la population locale dans ses quartiers résidentiels fermés ${ }^{2}$ : chaque quartier garde alors un accès unique avec des contrôles d'entrées et de sorties ${ }^{3}$; les habitants n'ont plus droit de sortir de leur quartier résidentiel sauf exception ${ }^{4}$. Les livraisons de courses et de médicaments sont organisées par les agents des communautés de quartier.

\section{Phase 3 : le déconfinement progressif}

Le 20 mars, alors que $88,7 \%$ des quartiers résidentiels fermés ne présentent aucun cas de Covid-19, le CDMLC annonce le déconfinement partiel : les entreprises, usines et administrations reprennent progressivement leurs activités. Les habitants des quartiers résidentiels sans cas positif peuvent sortir travailler ou faire leurs courses. Les réseaux de bus et de métro reprennent respectivement à partir du 23 et du 25 mars. Le 8 avril, la ville de Wuhan entame le déconfinement général. Les transports interrégionaux sont rétablis. Néanmoins, le contrôle de la température corporelle à l'entrée et à la sortie des quartiers résidentiels fermés est maintenu.

1. En Chine, les quartiers résidentiels fermés (xiao qu) sont généralement des complexes résidentiels fermés et sécurisés, composés de plusieurs ou plusieurs dizaines d'immeubles.

2. Même s'il s'agit d'une mesure de confinement au sein du quartier résidentiel fermé, en réalité les habitants n'ont plus le droit de sortir de chez eux. Ils ne peuvent pas circuler librement à l'intérieur du quartier résidentiel.

3. Une partie des cadres fonctionnaires et membres du parti sont arrivés en renfort dans les quartiers résidentiels à Wuhan à partir du 11 février pour aider à gérer l'organisation des services de proximité (livraison de courses, etc.) et le contrôle à l'entrée là la sortie.

4. Les exceptions comprennent les cas suivants : professionnels médicaux et travailleurs autorisés ; un seul membre de chaque ménage peut sortir tous les trois jours pour faire des courses afin de subvenir aux besoins essentiels. 
Néanmoins, si les tests de dépistage du Covid-19 sont nécessaires pour pouvoir bénéficier de soins hospitaliers, la capacité de test est très limitée par rapport à la demande : le 24 janvier, il est possible de faire environ 600 tests PCR par jour à Wuhan, alors que « la ville » compte 10 millions d'habitants. Même si cette capacité est multipliée par 33 à la date du 5 février ${ }^{19}$, le test, une ressource extrêmement rare à Wuhan, devient un véritable instrument de rationnement face au manque de lits. Parallèlement, le 24 janvier, le CDMLC met en place un système pyramidal de gestion des lits : les communautés de quartier et les centres de services sanitaires qui leur sont rattachés sont chargés de recenser les cas suspects et de classer les demandes d'accès aux soins en fonction de la gravité des cas parmi leurs habitants. Les centres de services sanitaires des communautés de quartier n'accueillent pas de patients atteints du Covid-19, mais jouent un rôle dans le tri des cas : les agents administratifs, les médecins et les soignants se déplacent tous les jours chez les habitants de leur quartier et remplissent les formulaires exigés par leurs supérieurs hiérarchiques. Ces informations sont remontées aux sous-districts correspondants, puis aux districts et enfin regroupées au CDMLC. Ce dernier, disposant d'informations centralisées sur la disponibilité des lits en temps réel dans les hôpitaux de Wuhan, décide de l'attribution des tests et des lits parmi les communautés de quartier. L'admission des patients se décide donc à l'échelle de la ville, et est le fait des cadres administratifs et non pas des professionnels de santé hospitaliers.

Dans ce système de gestion des lits hospitaliers, les communautés de quartier jouent un rôle essentiel : elles sont en charge de récolter des renseignements sur les habitants locaux (informations démographiques, informations épidémiologiques, etc.), d'organiser la mise en quarantaine des patients confirmés ou suspects, de surveiller le confinement, de désinfecter les quartiers, etc. Sur ce point, Wuhan n'est pas un cas isolé. Au contraire, quelle que soit la ville, les gouvernements municipaux s'appuient largement sur les communautés de quartier pour les renseignements et la gestion de la population locale (Liu, Chen, Wang, Xia, 2020 ; He, 2020).

\section{Une volonté de réduire les inégalités sociales d'accès aux soins mais un bilan mitigé}

En matière de réduction des inégalités sociales, la ville de Wuhan est pionnière en Chine. Dès le 21 janvier, le CDMLC de Wuhan annonce la prise en charge intégrale des frais de soins dus au Covid-19 par l'assurance maladie et par les gouvernements municipaux et provincial. En effet, selon les données communiquées par Xianjun Xiong, haut responsable de l'Administration nationale, jusqu'au 6 avril 2020, la moyenne du coût des soins hospitaliers pour les patients atteints du Covid-19 s'élève à 21500 yuan (soit 2687 euros), et pour ceux admis en soins intensifs ou en réanimation à 150000 yuan (soit 18800 euros) ${ }^{20}$. Selon

19. Avant le 22 janvier, seul le Centre de contrôle et de prévention des maladies (CCPM) provincial était autorisé à effectuer les tests PCR. Les hôpitaux de Wuhan doivent envoyer les échantillons prélevés au CCPM provincial qui a une capacité journalière de test très limitée. L'Administration municipale de santé de Wuhan autorise 9 hôpitaux publics et quelques laboratoires privés à effectuer les tests PCR respectivement à partir du 22 janvier et du 28 janvier. Les laboratoires privés à Wuhan sont en capacité d'effectuer plus de 10000 tests PCR par jour début février.

20. http://www.ccdi.gov.cn/yaowen/202004/t20200411_215163.html. 
la même source, 66,6 \% des frais sont pris en charge par l'assurance maladie. En effet, comme nous l'avons mentionné dans la première partie, le taux de remboursement se différencie largement selon la branche et les catégories socioprofessionnelles : les fonctionnaires peuvent bénéficier d'une prise en charge de la quasi-totalité des frais de soins, alors que les paysans en paient en général plus de $50 \%$. La configuration du système de l'assurance maladie en Chine est source d'inégalités sociales en termes d'accès aux soins et de conséquences financières. Dans ce contexte, la décision de la ville de Wuhan contribue à réduire ces inégalités : la municipalité décide de financer le reste à charge pour tous les patients atteints du Covid-19 et devant être hospitalisés. Autrement dit, quel que soit leur statut (citadin ou paysan) et quel que soit leur métier, les patients n'ont aucun reste à charge à payer. Néanmoins, cette décision a de lourdes conséquences financières. Par exemple, jusqu'au 19 février, le financement de ce reste à charge coûte à la municipalité de Wuhan plus de 450 millions de yuan (soit 56,3 millions d'euros) ${ }^{21}$.

La politique de financement intégral du reste à charge par les gouvernements locaux (municipaux et provinciaux) est très vite généralisée à toute la Chine, suite à une décision du Conseil des affaires de l'État, le 22 janvier. Cette politique est sans doute appréciable du point de vue de la réduction des inégalités sociales entre les individus, mais elle contribue largement à l'aggravation de l'endettement de ces gouvernements locaux. Au final, ces derniers supportent plus de $70 \%$ des dépenses engagées dans les différentes actions de lutte contre le Covid-19 (construction de nouveaux hôpitaux, prise en charge des frais de soins, etc.) ${ }^{22}$.

Néanmoins, d'autres facteurs d'inégalités sociales sont identifiés pendant l'épidémie de Covid-19, notamment dans la ville de Wuhan. D'abord, le rationnement et la distribution administrative des tests PCR et des lits dans les hôpitaux créent des inégalités dans l'accès aux soins hospitaliers, notamment pendant la période du 23 janvier au 16 février. En effet, les lits de soins intensifs sont progressivement créés, alors que la demande est en croissance exponentielle en continu. Le quota attribué à chaque communauté de quartier est très limité. Par exemple, selon notre entretien avec un agent administratif d'un sous-district, les communautés de quartier de son territoire obtiennent entre 3 et 10 lits d'hospitalisation par jour à la fin janvier, alors que la demande est 20 fois plus importante. En conséquence, les agents des communautés de quartier encouragent les habitants à mobiliser leurs propres réseaux pour être admis à l'hôpital. Entre le 20 janvier et le 16 février, de nombreux messages de demande d'aide sur les réseaux sociaux chinois (Weibo, Wechat, etc.) sont publiés. Les habitants locaux appellent aussi les différents numéros verts mis en place par la mairie de Wuhan et d'autres administrations municipales. Mais selon le même enquêté, les patients bénéficiant de réseaux politiques peuvent trouver un lit plus rapidement que ceux qui disposent de peu de réseaux, et qui

21. http://www.gov.cn/xinwen/gwylflkjz23/wzsl.htm. Les gouvernements locaux, et notamment la ville de Wuhan, récoltent 4,8 milliards de yuan (soit 602 millions d'euros) de dons monétaires via les fédérations de charité municipales.

22. http://czj.wuhan.gov.cn/BMDT/GZDT/202004/t20200422_1072558.html. 
sont obligés d'attendre pendant des jours ou des semaines.

Ensuite, la gestion territoriale de la lutte contre le Covid-19 entraîne des inégalités sociales entre les différents territoires. En janvier, les médias chinois se focalisent surtout sur l'épidémie dans la ville de Wuhan. Cependant, d'autres villes de la même province sont aussi gravement touchées par l'épidémie, notamment Xiaogan et Huanggang qui sont respectivement les deuxième et troisième villes les plus affectées de toute la Chine ${ }^{23}$. Mais pendant tout le mois de janvier, très peu de reportages ou de discussions sur ces deux villes en font état. La différenciation d'exposition médiatique semble avoir une forte corrélation avec le montant des transferts financiers de l'État central et les dons sociaux monétaires ou en nature effectués. Par exemple, selon les données publiées par la Fédération de charité provinciale de Hubei le 28 janvier, le total des dons monétaires perçus par la seule ville de Wuhan équivalait à l'addition des dons perçus respectivement par Xiaogan et par Huanggang 24 .

Enfin, les personnes atteintes d'autres maladies que le Covid-19 se retrouvent dans des situations particulièrement difficiles. Ceci est notamment le cas chez les individus souffrant de maladies chroniques et chez les personnes âgées à Wuhan. Comme expliqué plus haut, plusieurs hôpitaux de catégorie III sont transformés en établissement d'accueil des malades du Covid-19. Les patients non-Covid-19 déjà sur place sont transférés vers d'autres établissements (souvent plus petits et moins équipés) ou renvoyés à domicile. Selon notre enquête auprès des agents de communautés de quartier à Wuhan, bien que l'Administration nationale de santé ait publié une circulaire demandant aux centres de soins de chaque communauté de quartier d'assurer le suivi des malades chroniques ${ }^{25}$, les médecins et soignants sont dans l'incapacité de leur garantir les soins, car ils sont concentrés sur la lutte contre le Covid-19. De nombreux récits de vie et appels à l'aide publiés par les habitants sur les réseaux sociaux chinois témoignent des difficultés rencontrées par ces patients dans l'accès aux soins.

\section{Gestion des pénuries de matériels et mobilisation de l'industrie}

Pendant l'épidémie, au-delà du manque de lits, Wuhan doit faire face à des pénuries de matériels médicaux (respirateurs artificiels, oxygène médical, etc.) et d'équipements de protection individuelle (masques, vêtements de protection, gants chirurgicaux, etc.) pendant un mois et demi entre mi-janvier et fin février. Des milliers de messages d'appel aux dons

23. Ces deux villes enregistrent respectivement 3518 et 2905 cas de Covid-19 à la date du 29 février. Xiaogan (4,9 millions d'habitants) et Huanggang (6,3 millions d'habitants) comptent au total le même nombre d'habitants que Wuhan (11 millions). Bien que les taux d'incidence du Covid-19 (nombre de cas rapportés à la population pour 100000 habitants) dans ces deux villes soient moins importants qu'à Wuhan, elles sont beaucoup moins bien dotées en lits d'hôpitaux, notamment en lits de réanimation. En effet, on constate une concentration des services hospitaliers à Wuhan parmi les 13 villes-préfectures à Hubei : plus de $35 \%$ des meilleurs hôpitaux (67 hôpitaux en catégorie III et IIIA) se trouvent à Wuhan. Xiaogan et Huangang n'ont que respectivement 10 et 8 hôpitaux des mêmes catégories. On observe un manque de lits hospitaliers et de matériels médicaux dans ces deux villes.

24. https://www.hbcf.org.cn/ppv.html?ppvld=93efd338-b4a8-4620-952c-a0135aa59622.

25. http://www.nhc.gov.cn/jkj/s3577/202001/dd1e502534004a8d88b6a10f329a3369.shtml. 
sont publiés chaque jour par les hôpitaux et les professionnels de santé de la ville, et relayés par les internautes sur les réseaux sociaux, notamment sur le site de Weibo (« microblog ", équivalent de Twitter en Chine) entre le 20 janvier et le 10 février. Ces messages sont souvent accompagnés de photographies de masques faits maison, de surblouses artisanales fabriquées à partir des sacs-poubelle ainsi que de récits expliquant le manque d'équipements d'assistance respiratoire pour témoigner de l'urgence de la situation.

\section{Appels à la solidarité et gestion bureaucratique des dons}

Dans ces circonstances, le gouvernement municipal réagit assez lentement : le 23 janvier, le CDMLC de Wuhan annonce que le stock de matériels médicaux et d'équipements de protection est suffisant et qu'il n'y a aucun motif d'inquiétude. Ce discours est critiqué le soir même par une émission de télévision de la chaîne nationale (« CCTV News ») qui relaye les messages d'appel aux dons des soignants. Le lendemain, le CDMLC lance un appel aux dons en précisant leur gestion centralisée par deux associations parapubliques 26 : tous les dons monétaires et de matériels non médicaux seront récoltés par la Fédération de Charité de Wuhan ; les dons de matériels médicaux et de protection seront collectés par la Croix-Rouge locale. Ces dons seront ensuite redistribués par ces deux structures vers les hôpitaux. Les hôpitaux n'ont donc plus le droit de recevoir des dons directs venant d'individus ou de sociétés.

La gestion monopolistique des dons par ces deux structures semi-publiques fait l'objet de nombreuses et sévères critiques. En effet, les entreprises et individus chinois se mobilisent très vite, dès le 20 janvier, pour envoyer des dons monétaires ou en nature à Wuhan. De nombreux groupes de solidarité de différentes tailles sont créés sur Wechat (équivalent de WhatsApp) pour partager les informations (appels aux dons, donateurs éventuels, etc.), récolter les dons monétaires et en nature et les transporter à Wuhan. Par exemple, la Fédération de Charité de la ville reçoit 4,8 milliards de yuan de dons (soit 600 millions d'euros). Des centaines de milliers de masques et d'autres équipements de protection sont envoyés par les Chinois de toutes les régions. Cependant, les hôpitaux de Wuhan continuent de dénoncer le manque de matériel sur les réseaux sociaux. En effet, selon nos entretiens avec les bénévoles responsables de deux groupes de solidarité ayant affaire avec la CroixRouge de Wuhan, les dons en nature (matériels médicaux et de protection) sont obligés de passer par son intermédiaire alors même qu'elle n'a pas la capacité de gérer une telle quantité de matériel. D'une part, la Croix-Rouge de Wuhan ne compte que 13 titulaires et une cinquantaine de bénévoles jusqu'au 25 janvier 2020 ; d'autre part, la gestion du stockage et de la redistribution des dons n'est pas informatisée : tout est renseigné sur papier. Le $1^{\text {er }}$ février, des journalistes de la CCTV pointent l'inefficacité de la Croix-Rouge de Wuhan en publiant des photos de stades ou de centres d'exposition complètement remplis de matériels médicaux et de protection, qui sont censés être redistribués aux hôpitaux depuis plus de 10 jours. Dès le 2 février, le CDMLC supprime ce système de gestion monopolistique des dons : les hôpitaux peuvent désormais recevoir des dons directement auprès des donateurs.

26. Dans la littérature anglophone sur les ONG, ce type d'associations est appelé Governmentorganized non-governmental organization (GONGO). Pour plus de précisions, voir Pech (2011). 


\section{Mobilisation du secteur industriel}

À partir du 28 janvier, face aux pénuries continues de matériels médicaux et de protection, le gouvernement central et la municipalité de Wuhan commencent également à mobiliser le secteur industriel 27. Les entreprises nationales implantées à Wuhan sont d'abord sollicitées. Par exemple, le maire de Wuhan rencontre le directeur général de Wuhan Iron and Steel le 28 janvier, lui demandant d'augmenter sa capacité de production d'oxygène médical et d'aider les hôpitaux à installer des systèmes d'oxygène dans les meilleurs délais ${ }^{28}$. Les entreprises nationales d'autres régions sont également convoquées par le gouvernement central pour mieux faire face aux pénuries à Wuhan. Par exemple, le ministère de l'Industrie et des Technologies de l'information chinois alloue une vingtaine de milliers d'équipements de protection fabriqués en urgence à la fin janvier par une entreprise nationale dans la province de Shandong. Dans les communiqués de presse, les gouvernements à différents niveaux emploient le terme de «négociation » pour décrire la mobilisation de l'industrie. Mais selon le professeur et conseiller du maire de Pékin interviewé, ces entreprises nationales n'ont pas vraiment le choix face à cette demande, car les rapports avec les gouvernements constituent un enjeu très important pour la survie de leur activité.

Au-delà de la mobilisation des entreprises publiques, le gouvernement central met également en place des mesures spécifiques pour inciter à la production des matériels médicaux et d'équipements de protection. En effet, beaucoup d'usines chinoises ont fermé leurs portes entre le 24 janvier et le début du mois de février pour la fête de Nouvel An chinois. Le gouvernement central promet donc des primes pour financer les surcoûts liés à l'augmentation de la capacité productive pendant les jours fériés, ainsi que l'achat par l'État des produits non vendus en cas de surproduction. À la suite de ces annonces, un nombre important d'usines privées se lancent dans la fabrication de matériels de protection ${ }^{29}$. Dans le secteur du transport, plusieurs entreprises privées se mobilisent en créant des lignes spécifiques pour les transporter en priorité et

27. Wuhan est une mégapole industrielle. Les industries optoélectronique et informatique, manufacturière (notamment automobile), pharmaceutique et biomédicale sont les piliers de l'économie locale. Selon l'« Annuaire statistique de Wuhan 2018 », les revenus totaux de ces trois secteurs dépassent 500 milliards de yuan (soit 62,5 milliards d'euros).

28. Dans la province de Hubei, l'un des principaux producteurs d'oxygène médical est une filiale de Wuhan Iron and Steel : elle fournit $50 \%$ de l'oxygène médical dans cette province.

29. Selon une enquête réalisée par une équipe d'économistes l'Université de Wuhan, dans cette ville, la situation des entreprises industrielles pendant la propagation du Covid-19 se différencie selon la branche du métier et la taille de l'entreprise (Luo et al., 2020). Le congé du Nouvel An chinois est prolongé jusqu'au 13 février dans la province de Hubei suite à une décision prise par le Conseil des affaires de l'État le 26 janvier. À Wuhan, plus de $90 \%$ de PME et d'entreprises d'automobiles mettent à l'arrêt leurs activités dès le 23 janvier, leurs travailleurs étant souvent des ouvriers migrants habitués à retourner dans leur région natale pour fêter le Nouvel An. Ces entreprises ne peuvent réouvrir leurs portes avant la mi-mars, car celles qui souhaitent reprendre les activités économiques pendant le confinement doivent obtenir une autorisation de la part du CDMLC. Cependant, d'autres producteurs ne connaissent pas la même situation : les grandes entreprises (publiques ou privées) optoélectroniques et informatiques, pharmaceutiques et biomédicales, de la métallurgie et de la construction ne ferment pas leurs portes ni pendant le congé du Nouvel An ni pendant le confinement, même si la capacité de production est maintenue à un niveau plus faible qu'en temps normal. Beaucoup de ces entreprises sont mobilisées par le CDMLC pour fabriquer des matériels médicaux et des équipements de protection. 
gratuitement vers la province de Hubei. Néanmoins, la mobilisation volontaire des entreprises privées est beaucoup moins médiatisée et moins reconnue par les gouvernements chinois que celle des entreprises publiques. De manière générale, on peut dire que le gouvernement chinois central et le gouvernement local ont sous-estimé la gravité des pénuries de matériels en début d'épidémie, car la mairie de Wuhan a simplement compté sur les dons en lançant des appels à la solidarité. C'est seulement à partir de la fin janvier 2020 que les pouvoirs publics central et municipal commencent à mobiliser le secteur de l'industrie, ce qui ne facilite pas la gestion des pénuries de matériels et la lutte contre le Covid-19.

\section{Conclusion}

Dans cet article, nous avons analysé à partir d'une enquête par entretiens et d'études d'archives publiques ou transmises par nos enquêtés la mobilisation sanitaire de l'État chinois face à l'épidémie de Covid-19 entre janvier et mars 2020, avec une focalisation sur la ville de Wuhan, épicentre de la pandémie. Le gouvernement municipal (et en particulier le Comité de direction municipal pour la lutte contre le Covid-19 de Wuhan) joue un rôle clé durant cette période de crise sanitaire. D'une part, les décisions importantes sont principalement prises à cette échelle, en s'appuyant sur les conseils d'un comité scientifique : la construction et la transformation des hôpitaux, la centralisation de l'information sur la disponibilité des lits ainsi que la redistribution administrative des tests et des lits par le CDMLC, la mobilisation des entreprises nationales implantées dans la ville pour augmenter la production des équipements médicaux, etc.
D'autre part, les dépenses liées à la lutte contre le Covid-19, y compris les primes versées aux professionnels de santé mobilisés, sont majoritairement supportées par les gouvernements locaux, notamment la municipalité. Quant à l'État central, il joue un rôle important mais secondaire : il assure la coordination de la mobilisation interrégionale des professionnels de santé et du matériel, la mobilisation de l'industrie et notamment des entreprises nationales pour la production des équipements, et le financement d'une partie des dépenses liées à la lutte contre le Covid-19.

Au final, il nous semble donc réducteur de parler d'une mobilisation sanitaire de l'État chinois de manière globale comme le font la plupart des médias et certains travaux scientifiques : il est plus pertinent de parler des mobilisations sanitaires de la part des pouvoirs publics à différents niveaux. Ce régime de mobilisation, s'appuyant sur la municipalité comme pilier principal, présente des avantages (notamment, le rapprochement des décisions politiques du terrain) mais pose également plusieurs problèmes. Premièrement, les provinces et les municipalités des différentes régions n'ont pas les mêmes capacités de mobilisation. Comme nous l'avons montré dans cet article, les grandes villes comme Wuhan attirent plus d'attention politique et médiatique, et elles reçoivent davantage d'aides publiques et de dons, ce qui creuse les inégalités économiques, financières et sociales entre les différents territoires. Deuxièmement, les municipalités ne sont pas seulement les responsables politiques, mais également les responsables financiers de l'action publique. Malgré les recettes fiscales et les dons perçus par les organismes de charité à l'échelle locale, les coûts (en particulier le manque à gagner des recettes fiscales) 
et les dépenses liées à la lutte contre l'épidémie de Covid-19 ont aggravé l'endettement des gouvernements locaux, notamment dans les régions les plus pauvres de la Chine.

Achevé de rédiger le 9 octobre.

\section{Sources :}

Audin J. (2015), « Gouverner par la communauté de quartier (shequ) en Chine : ethnographie de la bureaucratisation participante des comités de résidents à Pékin ", Revue française de science politique, vol. $65, n^{\circ} 1$, p. 85 110, https://doi.org/10.3917/rfsp.651.0085.

Bray D. (2006), « Building "Community": New Strategies of Governance in Urban China ", Economy and Society, vol. $35, n^{\circ} 4$, p. 530-549, https://doi.org/10.1080/03085140600960799.

Chen S., Zhang Z., Yang J., Wang J., Zhai X., Bärnighausen T., Wang C. (2020), "Fangcang shelter hospitals: A novel concept for responding to public health emergencies ", The Lancet, vol. 395, $\mathrm{n}^{\circ} 10232$, p. 13051314, https://doi.org/10.1016/S0140-6736(20) 30744-3.

Gao X., Yu J. (2020), « Public governance mechanism in the prevention and control of the COVID-19: Information, decision-making and execution ", Journal of Chinese Governance, vol. 5 , n ${ }^{\circ} 2$, p. 178-197, https://doi.org/10.1080/ 23812346.2020.1744922

Gu E., Li L. (2020), « Crippled community governance and suppressed scientific/ professional communities: A critical assessment of failed early warning for the COVID-19 outbreak in China ", Journal of Chinese Governance, vol. 5, no 2, p. 160-177, https:// doi.org/10.1080/23812346.2020.1740468.

He M. (2020), " Le rôle des communautés de quartier dans la lutte contre la Covid-19 à Chengdu », Colloque "Covid-19 au prisme de la sociologie » [en ligne], Tsinghua University, Pékin, Chine, 26 avril [en chinois].

Jia K., Zhao Q (2008), 30 ans de réformes économiques chinoises : finances publiques et politiques budgétaire (1978-2008), Presse de Chongqing Université [en chinois].

Jiang Y., Yang J. (2015), « Route of achieving hierarchical medical system by optimizing the elements of affecting the choice of medical service ", Chinese Hospitals, vol. 19, n 12 , p. 15-17 [en chinois].
Li Y., Yao D., Li J., Zhu H., Chen Y. (2020), "Study on medical service pricing method in public hospital ", Soft Science of Health, vol. $34, n^{\circ} 3$, p. 23-26 [en chinois].

Li Z. (2020), « Research on the problems and countermeasures of the market-orientated reform of drug prices in China ", Strait Pharmaceutical Journal, $n^{\circ} 4$, p. 224-227 [en chinois].

Lian Y. (2019), " Drug reform. From the last few kilometers to the last mile ", Chinese Health, $n^{\circ}$ 8, p. 46-49 [en chinois].

Liu Y., Qi L., Zhu Q. et al. (2014), « History and relevant policies of the private medical institutions in China », Chinese Hospital Management, vol. $34, n^{\circ} 5$, p. 14-17 [en chinois].

Liu P., Zhong X., Yu S. (2020), « Striking a balance between science and politics: Understanding the risk-based policy-making process during the outbreak of COVID-19 epidemic in China ", Journal of Chinese Governance, vol. 5, n' 2, p. 198-212, https://doi.org/10.108 0/23812346.2020.1745412.

Liu S., Chen J., Wang Y., Xia D. (2020), « Sociological thinking of epidemic prevention and control ", Academics, $n^{\circ}$ 2, p. 50-67 [en chinois].

Luo Z., Sheng H., Li X. et al. (2020), « Entreprises de la province de Hubei face au Covid-19 », [en ligne] https://www.thepaper. cn/newsDetail_forward_6238948 [en chinois].

Meng Q., Fang H., Liu X., Yuan B., Xu J. (2015), "Consolidating the social health insurance schemes in China: Towards an equitable and efficient health system ", The Lancet, vol. 386, $n^{\circ} 10002$, p. 1484-1492, https://doi. org/10.1016/S0140-6736(15)00342-6.

Pech T. (2011), « Les nouvelles frontières de l'humanitaire ", in Paugam S. (dir.), Repenser la solidarité, Paris, Puf, p. 783-795, https://doi. org/10.3917/puf.pauga.2007.02.0783.

Wang S. (2020), "Wuhan, vivre et survivre ", La Vie des idées, https://laviedesidees.fr/ Wuhan-vivre-et-survivre.html.

Wei X. (2020), « China's response to the coronavirus pandemic », Cambridge Core Blog, https://bit.ly/34cY2nb.

Zhang Q. (2011), " Décentralisation fiscale en Chine : expériences, défi et nouveau chemin ", Tribune of Political Science and Law, $n^{\circ}$ 5, p. 94-101 [en chinois].

Zhang Z., Li Y. (2015), « Development and reform of the legal system for health insurance 
in China ", Chinese Journal of Health Policy, vol. $8, n^{\circ} 4$, p. 35-40 [en chinois].

Zhou X. (2017), The Institutional Logic of Governance in China: An Organizational Approach, SDX \& Harvard-Yenching Academic Library.
Zhou F. (2006), « A decade of tax-sharing: The system and its evolution "Social Science in China, $\mathrm{n}^{\circ}$ 6, p. 100-115 [en chinois].

Zhou F. (2012), Benefiting from Profit: Fiscal Relation and Behaviour of Local Government, Joint Publishing [en chinois]. 